\title{
The effect of intracortical bone pin application on \\ kinetics and tibiocalcaneal kinematics of walking gait
}

Christian Maiwald

Institute of Human Movement Science and Health, Technische Universität Chemnitz, Chemnitz, Germany

Anton Arndt

The Swedish School of Sport and Health Sciences (GIH), Stockholm, Sweden, and Karolinska Institute, Stockholm, Sweden

Chris Nester,

School of Health Sciences, University of Salford, Salford, UK

Richard Jones,

School of Health Sciences, University of Salford, Salford, UK

Arne Lundberg,

Karolinska Institute, Stockholm, Sweden

Peter Wolf,

Sensory-Motor Systems Lab, ETH, Zurich, Switzerland

(C) 2016. This manuscript version is made available under the Elsevier user license http://www.elsevier.com/open-access/userlicense/1.0/ 


\section{Abstract}

Bone anchored markers using intracortical bone pins are one of the few available methods for analyzing skeletal motion during human gait in-vivo without errors induced by soft tissue artifacts. However, bone anchored markers require local anesthesia and may alter the motor control and motor output during gait. The purpose of this study was to examine the effect of local anesthesia and the use of bone anchored markers on typical gait analysis variables. Five subjects were analyzed in two different gait analysis sessions. In the first session, a protocol with skin markers was used. In the second session, bone anchored markers were added after local anesthesia was applied. For both sessions, three dimensional infrared kinematicsof the calcaneus and tibia segments, ground reaction forces, and plantar pressure data were collected. 95\% confidence intervals and boxplots were used to compare protocols and assess the data distribution and data variability for each subject. Although considerable variation was found between subjects, withinsubject comparison of the two protocols revealed non-systematic effects on the target variables. Two of the five subjects walked at reduced gait speed during the bone pin session, which explained the between-session differences found in kinetic and kinematic variables. The remaining three subjects did not systematically alter their gait pattern between the two sessions. Results support the hypothesis that local anesthesia and the presence of bone pins still allow a valid gait pattern to be analyzed.

\section{Keywords}

gaitanalysis; bone anchored markers; walking; kinematics; ground reaction forces; plantar pressures. 


\section{Introduction}

Over the past decades, marker-based optoelectronic stereophotogrammetry has become a standard procedure in many types of dynamic analysesof the lower extremity and the footduring human locomotion. However, the limitations of skin based marker placement (SKIN), especially the presence of soft tissue artifacts, are well-known [1]. Bone anchored markersusing intracortical bone pins (PIN) are considered to be one of the few available methodsfor analyzing skeletal motion of the lower extremityin-vivo without errors induced by soft tissue artifacts. Another appealing advantage of PIN over SKIN is the fact that bony structuresof the foot and ankle can be analyzed that are either too small for valid marker placement, suffer from heavy skin movement artifacts, or are inaccessible by surface markers, e. g. the navicular or the talus.Other techniques of image-based kinematic analyses, like magnetic resonance imaging,radiostereometric analysis or fluoroscopy, are currently only capable of capturing static or slow dynamic situations and thus may not be feasible for analyzing human locomotion.

The most evident drawback of PIN is the requirement of local anesthesia and its potential effects on motor control and motor output. Previous studies have demonstrated effects of local anesthesia on roll-over during gait, e. g. the increase of metatarsal head loading in plantar pressure data after applying ice to the plantar surface of the foot[2]. Furthermore, the mechanical intervention of protruding pins anchored to the bone might interfere with the motion of tendons, ligaments, skin and other pins applied in close proximity. Psychological effects of PIN cannot be ruled out either, as subjects may alter their gait patterns towards a more cautious roll-over pattern and less range of motion.

This study aimed to analyze whether the application of PIN results in altered motor output and thus, whether PIN is a valid method for analyzing human gait. The validity of PIN can only be assured if the magnitude and variability of human gait kinematics and kinetics areunaffected by local anesthesia and pin application.

An experimental protocol was designed with PIN as an independent variable, and with 
kinematicsand kinetics collected as target variables. It was hypothesized that target variable magnitude and variability would not differ between use of PIN and SKIN protocols.

The outcome of this study is an important step to validate previous and future studies, in which a similar PINapproach is used to analyze kinetics and kinematics during human gait [3-10].

\section{Methods}

Five healthy male subjects participated in the study. Anthropometric data for each subject are listed in Table 1. A sixth subject had to be excluded due to problems with the application of the pins. Study procedures were approved by the local ethics committee of the Karolinska Institute, Huddinge, Sweden.

Insert Table 1 here

Subjects were analyzed during normal walking gait in laboratory conditions, collecting kinetics and kinematics of their movement patterns. Two measurement sessions were carried out for each subjectin the following sequence, either on the same day or on two consecutive days: during session 1 (SKIN), a markerset of skin-mounted markers was applied to the calcaneus and tibia of each subject. Session 2 (PIN) succeeded SKIN and was conducted after local anesthetics had been applied and intracortical bone pins had been inserted to the bony structures of the subjects' right foot. Hence, a combined markerset of SKIN and PIN markers was used in session 2 (see Figure 1).The locations of SKIN markers were marked directly on subjects' skin to ensure the reproducible placement of SKIN markers after pin insertion.

Insert Figure 1 here 
Each subject was asked to complete 9-12 SKIN and at least 20 PIN walking gait trials. Data were collected while subjects walked on a $9.5 \mathrm{~m}$ walkway at their self-selected pace.

Kinematics were recorded using a 12-camera infrared system (Qualisys, Gothenburg, Sweden) sampling at $240 \mathrm{~Hz}$. Kinetics were captured by a force plate (Kistler, Winterthur, Switzerland, 960 Hz, sync to Kinematics) and a pressure distribution platform (Emed, Novel, Munich, Germany, 50 $\mathrm{Hz}$ ), which were mounted flush with the walkway and approximately $1 \mathrm{~m}$ apart from each other in order to capture two consecutive foot strikes of the same foot.

Differences between SKIN and PIN were assessed by analyzing relative motion of the shank and heel. In both conditions, four markers were attached to the calcaneus (one lateral, one medial, two posterior) in such a way as to prevent artifacts from the motion of the calcaneal fat pad. The motion of the shank was captured using six markers (fibular head, tibial tuberosity, middle of tibia crest, both malleoli, and the lower lateral malleolus, see [11]for marker positions).Gaps in marker trajectories were interpolated up to a gap size of 10 frames (42ms) using a higher order polynomial fit. Trials featuring larger trajectory gaps were discarded.

The technical reference frame for each segment was set up during relaxed standing in a neutral foot position, i.e., foot length axis was in parallel to the sagittal plane. The segment origin was placed at the mean marker location of all segment markers, and axes were aligned parallel to the global reference frame. Segment motions were calculated by matching corresponding markers across frames according to the procedures described by [12].This approach is robust against small deviations of marker placement between sessions, and does not rely on any markers placed on specific anatomical locations. Relative motion of the heel versus the shank in anatomical planes was calculated by projecting the rotation of the heel around its helical axis onto the shank coordinate system, accounting for the instantaneous helical axis orientation.

The range of motion (ROM) in three anatomical planes during the stance phase of subjects' gait was calculated as the target variable in order to allow for simple interpretation and comparison of the obtained results to other studies [3,11,13]. The stance phase was determined by setting a $10 \mathrm{~N}$ 
threshold on the Kistler force plate. Since the EMED platform was not synchronized to the kinematic system, the second stance phase was detected based on a local extremum of the acceleration of the heel markers similar to an approach based on foot velocity described by [14].

Two local maxima (Fz2 and Fz4) and one local minimum (Fz3) [15,16], as well as their relative timing (Fz2_t, Fz3_t, Fz4_t), and ground contact time (GCT) were calculated from force plate data. Gait speed was calculated based on the distance between two consecutive foot strikes, which was determined from the average positions of all heel markers. Kinematic data and force plate data were processed using custom-written Matlabsoftware (The Math Works, Natick, USA).

Plantar pressures were recorded using the EMED AT platform (4 sensors/cm², $50 \mathrm{~Hz}$ ). Raw data were exported to ASCII and analyzed using custom-written Matlab software as described in [17], including the definition of anatomical regions (masks M1 through M9, seeFigure 3). For the analyses conducted in this study, the relative force-time integral (FTI) of each mask as well as the timing of heel-off during roll-over (HO) were calculated.FTI was calculated as relative impulse of each mask compared to the total impulse of the foot area. HO was determined as the relative point in stance phase, at which the cumulated force of the heel sensors dropped below $10 \%$ of the total maximum force recorded during the entire stance phase. Other studies have employed similar variables to analyze roll-over timing based on plantar pressure data [18].

Arch regions M4 and M5 were not considered for analysis in this study, as none of the subjects' feet had any deformity or pathology, and thus most of the loads in M4 and M5 were near or exactly zero for each stance phase.

Insert Figure 2 here

Due to the small sample size and the explorative nature of this study, we did not conduct any statistical testing.Instead, 95\%-confidence intervals (upper bound: up95, lower bound: lo95) for the mean of each variable and condition were calculated from sample data. Data distribution and 
variance were assessed graphically using boxplots.

Non-overlapping confidence bounds for SKIN and PIN indicate possible differences between the two conditions. The practical relevance of this difference was rated based on the repeatability of the respective variable, which- due to the small sample of our dataset - was synthesized from other studies involving larger samples. As long as differences between SKIN and PIN did not exceed the expected random fluctuations of repeated measurements of a variable, they were considered practically irrelevant [19].

\section{Results}

Technical problems caused missing values for some of the subjects (kinematics: trajectory gaps in S3, GRF: operator faults in S2 \& S4). Table 2contains the 95\%-confidence bounds for all variables. Non-overlapping bounds are shaded grey. Figure 3depictsthe distribution of GRF data as an example.

Insert Table 2 here

None of the collected variables indicated systematic differences between SKIN and PIN conditions across all subjects. Differences in GRF were aggregated for S1, and kinematic differences aggregated for S5. These cases will be discussed in the following section. All other differences appeared to occur randomly and in a non-systematic manner.

Insert Figure 3 here

\section{Discussion}

The aim of this study was to investigate the influence of intracortical bone pins on the kinematics and kinetics of walking gait. GRF, plantar pressures, and 3D kinematics were measured with and 
without intracortical bone pins applied to the subjects' foot and shank, and compared between conditions. We hypothesized that the presence of pins would not systematically alter subjects' gait patterns. Due to the invasive nature of bone pin application, only a small sample of five subjects was available for analysis.

The results of these five subjects do not indicate a systematic effect of intracortical bone pins on the gait pattern during walking. Subjects 1 and 5 effectively reduced their gait speed after pin application, which explains the reduced excursion of vertical GRF curves, especially at Fz4. The pattern of GRF changes observed in our data was similar across subjects (lower Fz2 and Fz4 \& larger Fz3 and GCT for PIN). This can be interpreted as a less pronounced foot strike and a more cautious stance phase in the PIN condition and may be directly linked to gait speed [20]. Relative timing of GRF variables was only marginally influenced by PIN.

Effects of PIN on kinematic data appear to be less uniform. Only the data of S5 indicate an increase of transversal and a decrease of sagittal ROM in the PIN condition. The remaining subjects' data indicate a substantial amount of variability across trials even within conditions. However, similar effects have been observed in other studies [11] and may mask smaller, but more systematic effects. Being smaller than the normal variation we assume these are incidental and do not compromise the validity of the kinematic data.

Plantar pressure distributions did not indicate any systematic effect between SKIN and PIN. Only the increase of medial forefoot loading in S3 corresponded to the interpretation of GRF data that subjects tend to demonstrate a more cautious gait pattern in PIN.

In summary, PIN induced a systematically decreased gait dynamic in one of our subjects (S1). Across all subjects, effects on kinetics were consistently lower than 5\% (20-30 N vertical GRF, 1\% ROP in timing variables, $1 \%$ of relative FTI). These effect magnitudes were considered subtle, compared to the random fluctuations expected from repeated measures in this type of experimental design[21,22]. The most prominent differences between SKIN and PIN occurred in kinematic data with up to $25 \%$ of $\mathrm{ROM}\left(\sim 2-3^{\circ}\right)$. In light of the more variable nature of kinematic data, the SKIN- 
PIN differences in our data were considered small, based on the amount of variation to be expected from repeated trialscollected with a similar protocol, but with only skin markers attached[11]. Therefore, we considered the observed differences in kinetics and kinematics to be practically irrelevant given the limiting factors of experimental protocols in laboratory gait analysis.

The size of the 95\%-confidence-intervals as well as the size of the box-plots indicate that SKIN and PIN conditions caused the same amount of variability in the recorded data, for most of the subjects and most of the collected variables. Based on this observation, we did not find evidence for a less reliable, less secure or less stable gait in the PIN condition.

Therefore, the data of our study support our hypothesis that there is no difference in gait between SKIN and PIN conditions. A similar result was already observed for slow running gait by[3]. Both studies support the assumption that intracortical bone pins and local anesthesia still allow for a valid gait pattern to be analyzed.

Moreover, the authors think that the increasing availability of studies on the reliability of classic gait analyses causes a more general problem with these kinds of protocols: biomechanical data collected during human locomotion contains substantial amounts of variability across repeated trials, which statistically override the smaller systematic effects that researchers aim to observe when they conduct studies involvinggait analyses. This phenomenon does not appear to be causally linked to invasive bone pin application. Gait variability appears to be a more generic feature of human motor performance, and becomesespecially problematic when gait analysis protocols are conducted in a laboratory environment with a limited number of foot strikes to be captured and analyzed [23].

In the past, studies involving intracortical bone pins have not been conducted with sample sizes that would allow the generalization of the observed effects. Since a non-random sample was recruited in our study, its scope is of a predominantly descriptive nature. External validity may seem limited with such protocols. However, the benefit of applying invasive bone pins can be found in their contribution to the inductive reconstruction of theories about the kinematic mechanisms of bony 
structures, e. g. the amount of bony movement within the upper and lower ankle joints during gait [3]. Results of bone pin studies may be unexpected at first and specific to a small group of individuals, but may in turn stimulate the evolution of theories.

\section{Conclusion}

The results of this study suggest that intracortical bone pins applied under local anesthesia do not alter subjects' gait patterns in a systematic way. Data of gait analysis protocols using intracortical bone pins should therefore be comparable to protocols using skin mounted markers.

\section{Acknowledgements}

The authors would like to thank Qualysis AB for providing the cameras for the study. Financial assistance was received from the Swiss National Science Foundation and the Karolinska Institute’s Research Fund. We would also like to thank Lisa C. Peterson for her linguistic assistance with the manuscript. 


\section{References}

[1] Leardini A, Chiari L, Della Croce U, Cappozzo A. Human movement analysis using stereophotogrammetry. Part 3. Soft tissue artifact assessment and compensation. Gait \& posture 2005;21(2):212-25.

[2] Nurse MA, Nigg BM. The effect of changes in foot sensation on plantar pressure and muscle activity. Clinical biomechanics (Bristol, Avon) 2001;16(9):719-27.

[3] Arndt A, Wolf P, Liu A, Nester C, Stacoff A, Jones R et al. Intrinsic foot kinematics measured in vivo during the stance phase of slow running. Journal of biomechanics 2007;40(12):2672-8.

[4] Stacoff A, Reinschmidt C, Nigg BM, Van Den Bogert, A J, Lundberg A, Denoth J et al. Effects of foot orthoses on skeletal motion during running. Clinical biomechanics (Bristol, Avon) 2000;15(1):54-64.

[5] Stacoff A, Reinschmidt C, Nigg BM, Van Den Bogert, A J, Lundberg A, Denoth J et al. Effects of shoe sole construction on skeletal motion during running. Medicine and science in sports and exercise 2001;33(2):311-9.

[6] Nester C, Jones RK, Liu A, Howard D, Lundberg A, Arndt A et al. Foot kinematics during walking measured using bone and surface mounted markers. Journal of biomechanics 2007;40(15):3412-23.

[7] Lundgren P, Nester C, Liu A, Arndt A, Jones R, Stacoff A et al. Invasive in vivo measurement of rear-, mid- and forefoot motion during walking. Gait \& posture 2008;28(1):93-100.

[8] Wolf P, Stacoff A, Liu A, Nester C, Arndt A, Lundberg A et al. Functional units of the human foot. Gait \& posture 2008;28(3):434-41.

[9] Liu A, Nester CJ, Jones RK, Lundgren P, Lundberg A, Arndt A et al. Effect of an antipronation foot orthosis on ankle and subtalar kinematics. Medicine and science in sports and exercise 2012;44(12):2384-91.

[10] Arndt A, Lundgren P, Liu A, Nester C, Maiwald C, Jones R et al. The effect of a midfoot cut in the outer sole of a shoe on intrinsic foot kinematics during walking. Footwear Science 
2013;5(1):63-9.

[11] Wolf P, List R, Ukelo T, Maiwald C, Stacoff A. Day-to-Day consistency of lower extremity kinematics during walking and running. Journal of applied biomechanics 2009;25(4):369-76.

[12] Challis JH. A procedure for determining rigid body transformation parameters. Journal of biomechanics 1995;28(6):733-7.

[13] Arndt A, Westblad P, Winson I, Hashimoto T, Lundberg A. Ankle and subtalar kinematics measured with intracortical pins during the stance phase of walking. Foot \& ankle international 2004;25(5):357-64.

[14] O'Connor CM, Thorpe SK, O'Malley MJ, Vaughan CL. Automatic detection of gait events using kinematic data. Gait \& posture 2007;25(3):469-74.

[15] Stüssi E, Debrunner HU. Parameter-Analyse des menschlichen Ganges. Biomedizinische Technik/Biomedical Engineering 1980;25(s1):222-4.

[16] Stacoff A, Diezi C, Luder G, Stüssi E, Kramers-de Quervain IA. Ground reaction forces on stairs: effects of stair inclination and age. Gait \& posture 2005;21(1):24-38.

[17] Maiwald C, Grau S, Krauss I, Mauch M, Axmann D, Horstmann T. Reproducibility of plantar pressure distribution data in barefoot running. Journal of applied biomechanics 2008;24(1):1423.

[18] Cock A de, Clercq D de, Willems T, Witvrouw E. Temporal characteristics of foot roll-over during barefoot jogging: reference data for young adults. Gait \& posture 2005;21(4):432-9.

[19] Maiwald C, Axmann D, Grau S. Measurement error in footwear research biomechanics. Footwear Science 2011;3(2):117-24.

[20] Chiu M, Wang M. The effect of gait speed and gender on perceived exertion, muscle activity, joint motion of lower extremity, ground reaction force and heart rate during normal walking. Gait \& posture 2007;25(3):385-92.

[21] Reed LF, Urry SR, Wearing SC. Reliability of spatiotemporal and kinetic gait parameters determined by a new instrumented treadmill system. BMC musculoskeletal disorders 
2013;14:249.

[22] Putti AB, Arnold GP, Cochrane LA, Abboud RJ. Normal pressure values and repeatability of the Emed ST4 system. Gait \& posture 2008;27(3):501-5.

[23] Oriwol D, Milani TL, Maiwald C. Methodological issues associated with the mean value of repeated laboratory running measurements. Footwear Science 2012;4(3):183-90. 
Table 1: Anthropometric data for all five subjects

\begin{tabular}{|c|c|c|c|c|}
\hline Subject & Age [y] & Height [m] & Weight [kg] & BMI $\left[\mathrm{kg}^{*} \mathrm{~m}^{-2}\right]$ \\
\hline S1 & 36 & 1.80 & 70 & 21.6 \\
\hline S2 & 57 & 1.83 & 94 & 28.1 \\
\hline S3 & 35 & 1.73 & 75 & 25.1 \\
\hline S4 & 38 & 1.82 & 112 & 33.8 \\
\hline S5 & 32 & 1.80 & 71 & 21.9 \\
\hline Mean (SD) & $39.6(10.0)$ & $1.80(0.04)$ & $84.4(18.2)$ & $26.1(5.1)$ \\
\hline
\end{tabular}


Table 2: Overview of GRF, kinematics, and plantar pressure data of all five subjects

\begin{tabular}{|c|c|c|c|c|c|c|c|c|c|c|c|c|c|}
\hline & & & & \multicolumn{2}{|c|}{ S1 } & \multicolumn{2}{|c|}{ S2 } & \multicolumn{2}{|c|}{ S3 } & \multicolumn{2}{|c|}{ S4 } & \multicolumn{2}{|c|}{ S5 } \\
\hline & & & & skin & pin & skin & pin & skin & pin & skin & pin & skin & pin \\
\hline \multirow{14}{*}{$\frac{\frac{\amalg}{\Upsilon 2}}{\circlearrowleft}$} & & \multirow{2}{*}{ Fz2 [N] } & up95 & 765.4 & 710.0 & & & 990.8 & 931.4 & & & 1000.4 & 962.7 \\
\hline & & & 1095 & 735.7 & 672.0 & & & 946.8 & 902.0 & & & 938.8 & 910.5 \\
\hline & & \multirow{2}{*}{ Fz2_t [\% ROP] } & up95 & 20.5 & 20.2 & & & 21.5 & 21.3 & & & 20.6 & 20.8 \\
\hline & & & 1095 & 19.0 & 19.1 & & & 20.5 & 19.6 & & & 19.6 & 19.6 \\
\hline & & \multirow{2}{*}{ Fz3 [N] } & up95 & 456.3 & 477.7 & & & 474.5 & 506.9 & & & 437.1 & 447.4 \\
\hline & & & 1095 & 440.6 & 457.0 & & & 447.5 & 490.6 & & & 397.8 & 409.7 \\
\hline & & \multirow{2}{*}{ Fz3_t [\% ROP] } & up95 & 47.0 & 44.5 & & & 50.2 & 48.1 & & & 50.0 & 48.2 \\
\hline & & & lo95 & 44.9 & 41.8 & & & 46.9 & 46.4 & & & 48.2 & 47.0 \\
\hline & & \multirow{2}{*}{ Fz4 [N] } & up95 & 740.3 & 709.1 & & & 862.3 & 843.1 & & & 880.0 & 825.9 \\
\hline & & & 1095 & 716.1 & 694.4 & & & 832.0 & 821.7 & & & 830.9 & 808.2 \\
\hline & & \multirow{2}{*}{ Fz4_t [\% ROP] } & up95 & 77.8 & 75.8 & & & 78.1 & 77.9 & & & 77.2 & 76.2 \\
\hline & & & 1095 & 76.6 & 74.7 & & & 76.6 & 77.2 & & & 76.0 & 75.4 \\
\hline & & \multirow{4}{*}{ ROM frontal $\left[^{\circ}\right]$} & up95 & 0.642 & 0.670 & & & 0.630 & 0.658 & & & 0.636 & 0.628 \\
\hline & & & 1095 & 0.624 & 0.655 & & & 0.620 & 0.640 & & & 0.622 & 0.606 \\
\hline \multirow{8}{*}{ 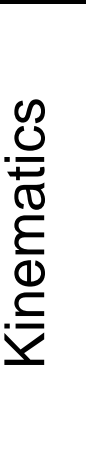 } & \multirow{8}{*}{ 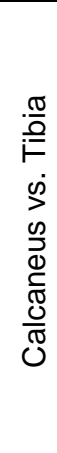 } & & up95 & 11.1 & 17.4 & 12.5 & 13.2 & & & 12.1 & 9.5 & 8.5 & 5.7 \\
\hline & & & 1095 & 9.5 & 8.8 & 10.6 & 8.9 & & & 9.3 & 8.5 & 6.3 & 4.9 \\
\hline & & \multirow{2}{*}{ ROM sagittal $\left[{ }^{\circ}\right]$} & up95 & 17.0 & 21.5 & 14.9 & 13.7 & & & 14.1 & 15.1 & 14.9 & 11.1 \\
\hline & & & 1095 & 15.3 & 14.5 & 12.0 & 12.4 & & & 10.3 & 13.8 & 13.1 & 10.2 \\
\hline & & \multirow{2}{*}{ ROM transversal $\left[{ }^{\circ}\right]$} & up95 & 9.5 & 13.2 & 9.6 & 8.8 & & & 11.4 & 9.4 & 6.8 & 10.3 \\
\hline & & & 1095 & 7.5 & 9.9 & 5.5 & 6.5 & & & 4.4 & 8.6 & 5.4 & 9.0 \\
\hline & & \multirow{2}{*}{ gait speed $[\mathrm{m} / \mathrm{s}]$} & up95 & 1.49 & 1.36 & 1.42 & 1.44 & & & 1.52 & 1.49 & 1.55 & 1.48 \\
\hline & & & lo95 & 1.42 & 1.28 & 1.38 & 1.38 & & & 1.45 & 1.43 & 1.51 & 1.45 \\
\hline \multirow{16}{*}{ 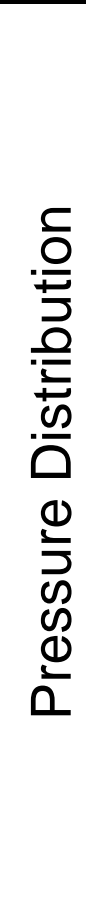 } & & \multirow{2}{*}{ HO [\% ROP] } & up95 & 67.9 & 67.9 & 75.0 & 63.5 & 54.5 & 53.6 & 66.2 & 59.9 & 53.6 & 53.7 \\
\hline & & & lo95 & 64.7 & 57.6 & 43.3 & 55.2 & 49.4 & 45.6 & 59.1 & 54.8 & 49.1 & 50.4 \\
\hline & & \multirow{2}{*}{ FTI M1 [\%] } & up95 & 23.3 & 24.3 & 25.5 & 22.2 & 20.3 & 20.8 & 20.3 & 19.7 & 21.9 & 21.5 \\
\hline & & & 1095 & 20.8 & 19.7 & 15.6 & 19.1 & 18.1 & 15.7 & 18.1 & 17.7 & 19.7 & 20.0 \\
\hline & & ETI M & up95 & 17.5 & 16.6 & 16.3 & 12.0 & 13.6 & 11.9 & 13.2 & 12.3 & 14.2 & 14.7 \\
\hline & & & lo95 & 15.7 & 10.3 & 6.8 & 10.5 & 11.4 & 8.7 & 11.9 & 10.4 & 12.5 & 13.2 \\
\hline & & [0/ & up95 & 4.1 & 5.6 & 5.7 & 3.7 & 3.6 & 3.0 & 5.8 & 4.8 & 1.0 & 0.7 \\
\hline & & & 1095 & 1.8 & 2.2 & 1.0 & 3.0 & 2.4 & 2.3 & 5.0 & 4.1 & 0.2 & 0.3 \\
\hline & & & up95 & 17.6 & 21.3 & 23.5 & 17.3 & 12.0 & 12.8 & 12.5 & 13.5 & 15.3 & 16.5 \\
\hline & & & 1095 & 13.3 & 16.8 & 7.2 & 14.5 & 10.0 & 10.2 & 11.0 & 11.3 & 9.5 & 12.6 \\
\hline & & FTIM & up95 & 31.6 & 35.3 & 34.6 & 31.2 & 27.5 & 30.3 & 29.4 & 31.3 & 36.3 & 35.0 \\
\hline & & ] & 1095 & 30.3 & 29.3 & 20.8 & 29.1 & 26.2 & 28.3 & 27.0 & 29.2 & 33.9 & 33.2 \\
\hline & & & up95 & 7.3 & 6.3 & 20.0 & 14.3 & 20.7 & 22.5 & 13.4 & 13.1 & 14.9 & 12.5 \\
\hline & & (1) & 1095 & 4.0 & 2.8 & 5.6 & 12.3 & 18.2 & 16.2 & 10.3 & 10.7 & 9.7 & 9.4 \\
\hline & & & up95 & 7.3 & 5.9 & 18.0 & 4.9 & 7.7 & 9.2 & 8.0 & 8.8 & 6.8 & 5.9 \\
\hline & & (1) & 1095 & 4.5 & 2.8 & 0.0 & 3.1 & 6.2 & 6.7 & 6.5 & 7.6 & 4.0 & 4.5 \\
\hline
\end{tabular}




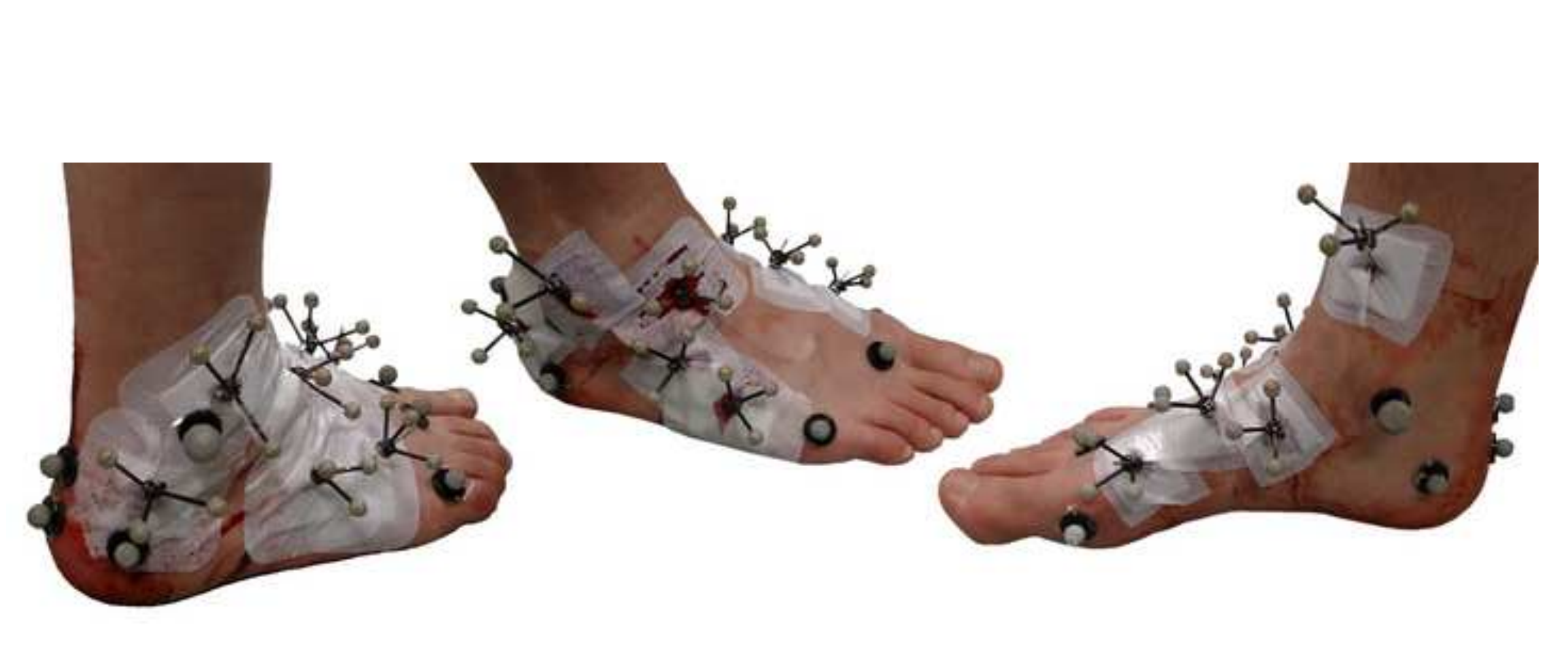

-

.

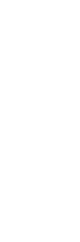




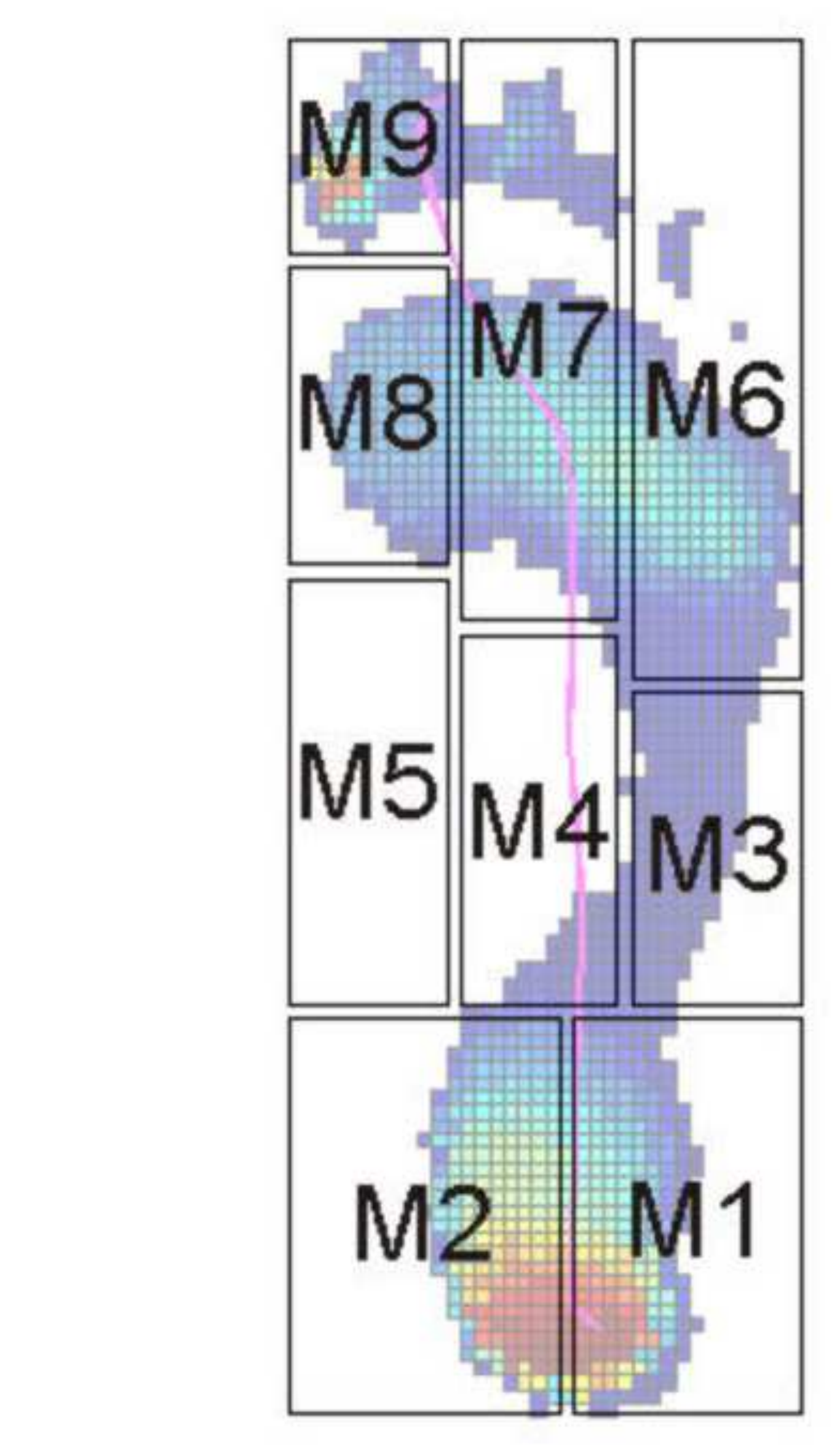

\section{Figure(s)}

)

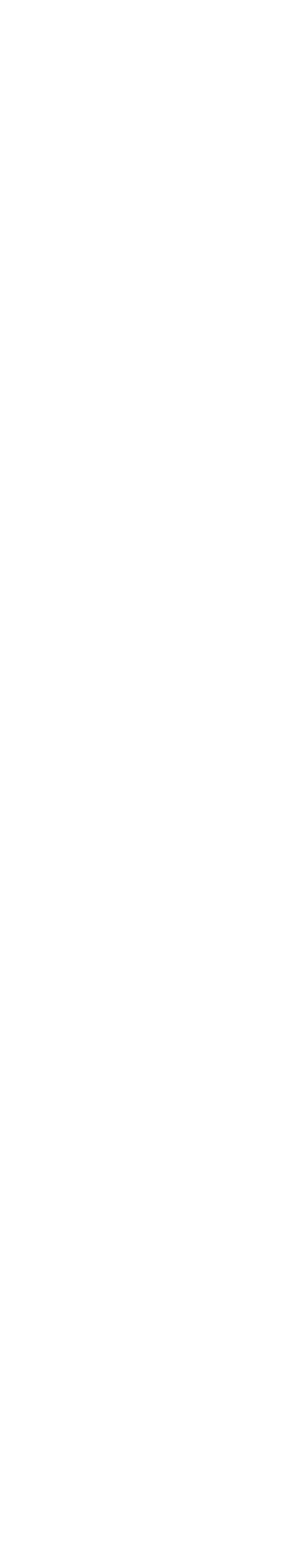


GRF: SKIN (blue) vs. PIN (red)

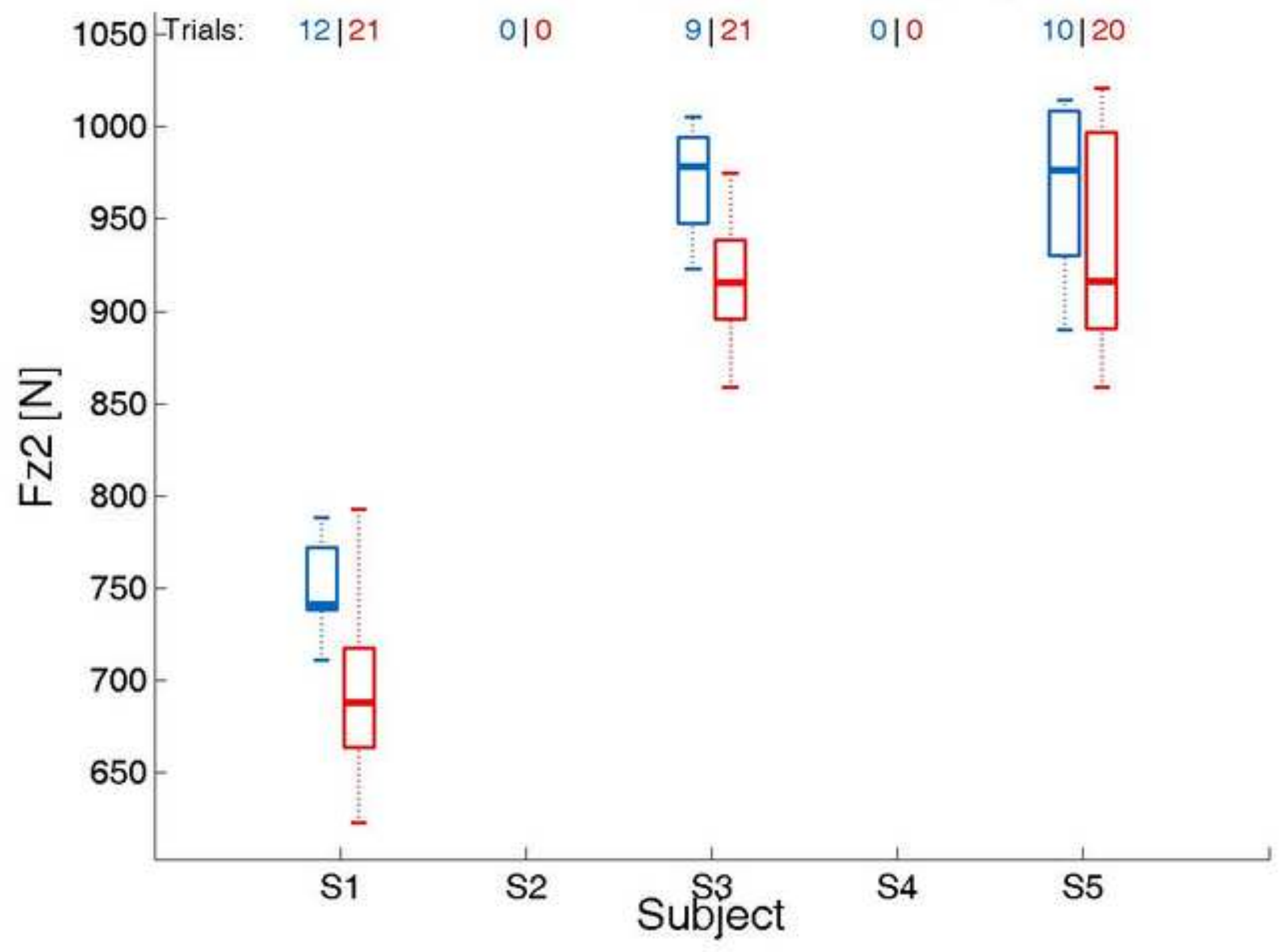


GRF: SKIN (blue) vs. PIN (red)

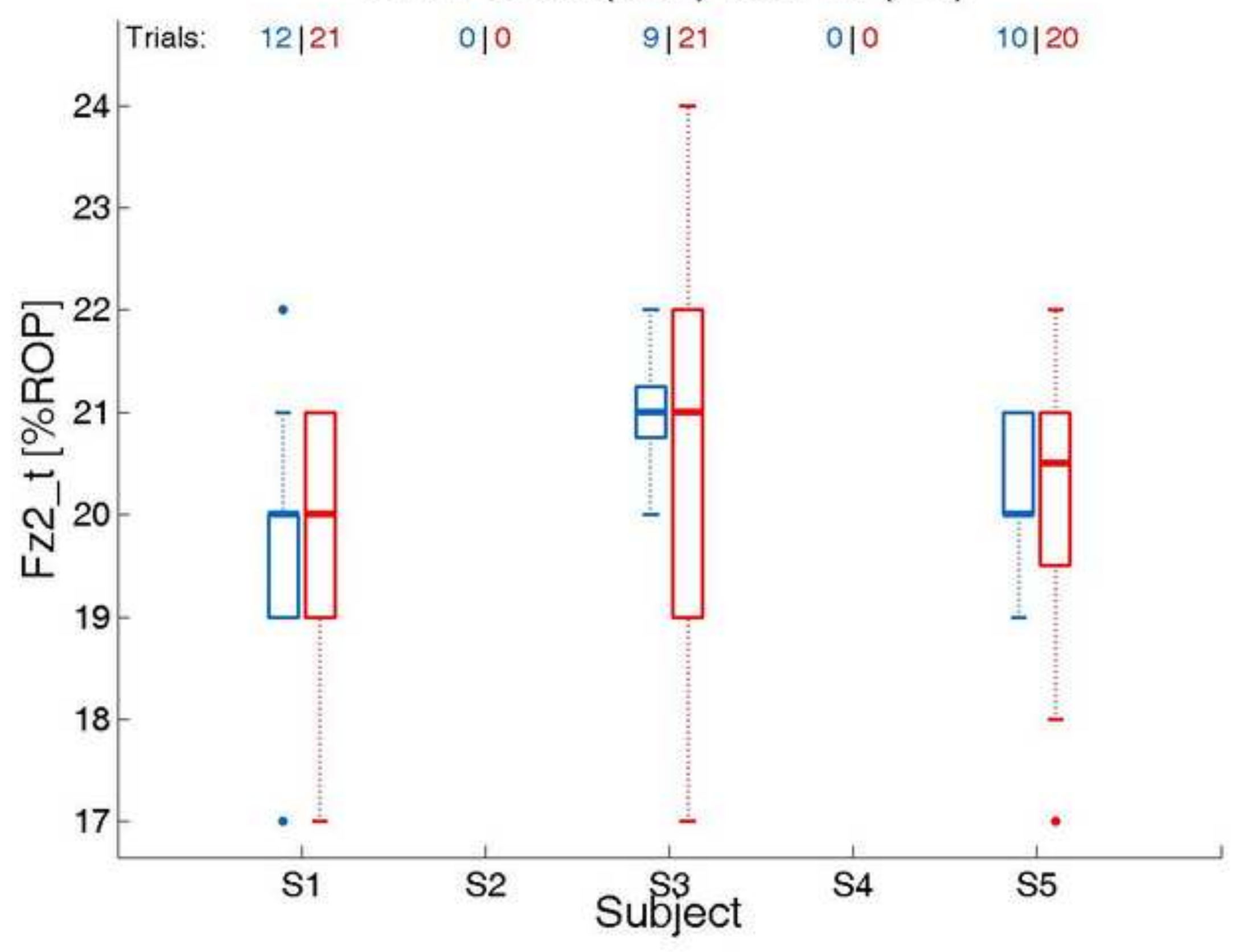


GRF: SKIN (blue) vs. PIN (red)

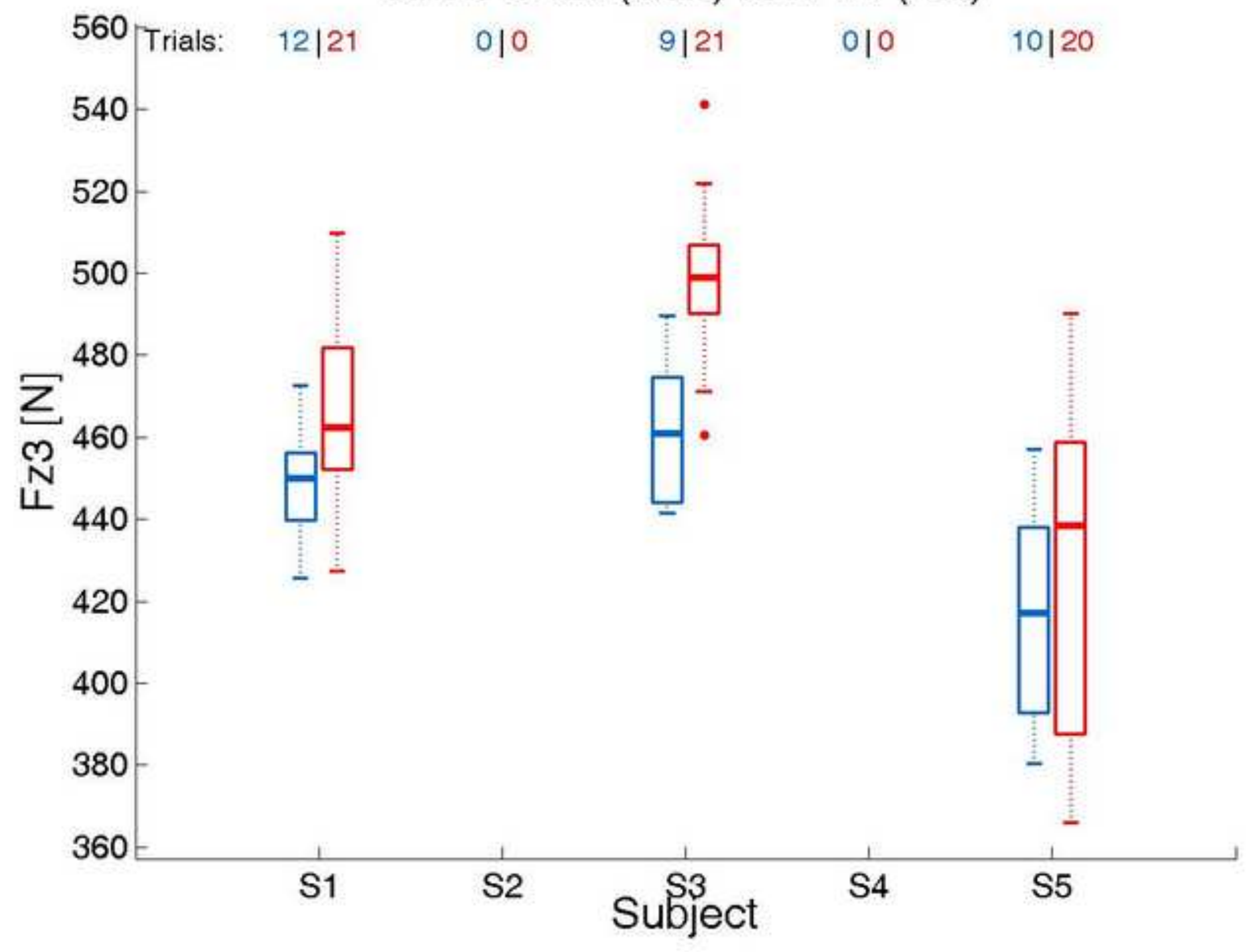


GRF: SKIN (blue) vs. PIN (red)

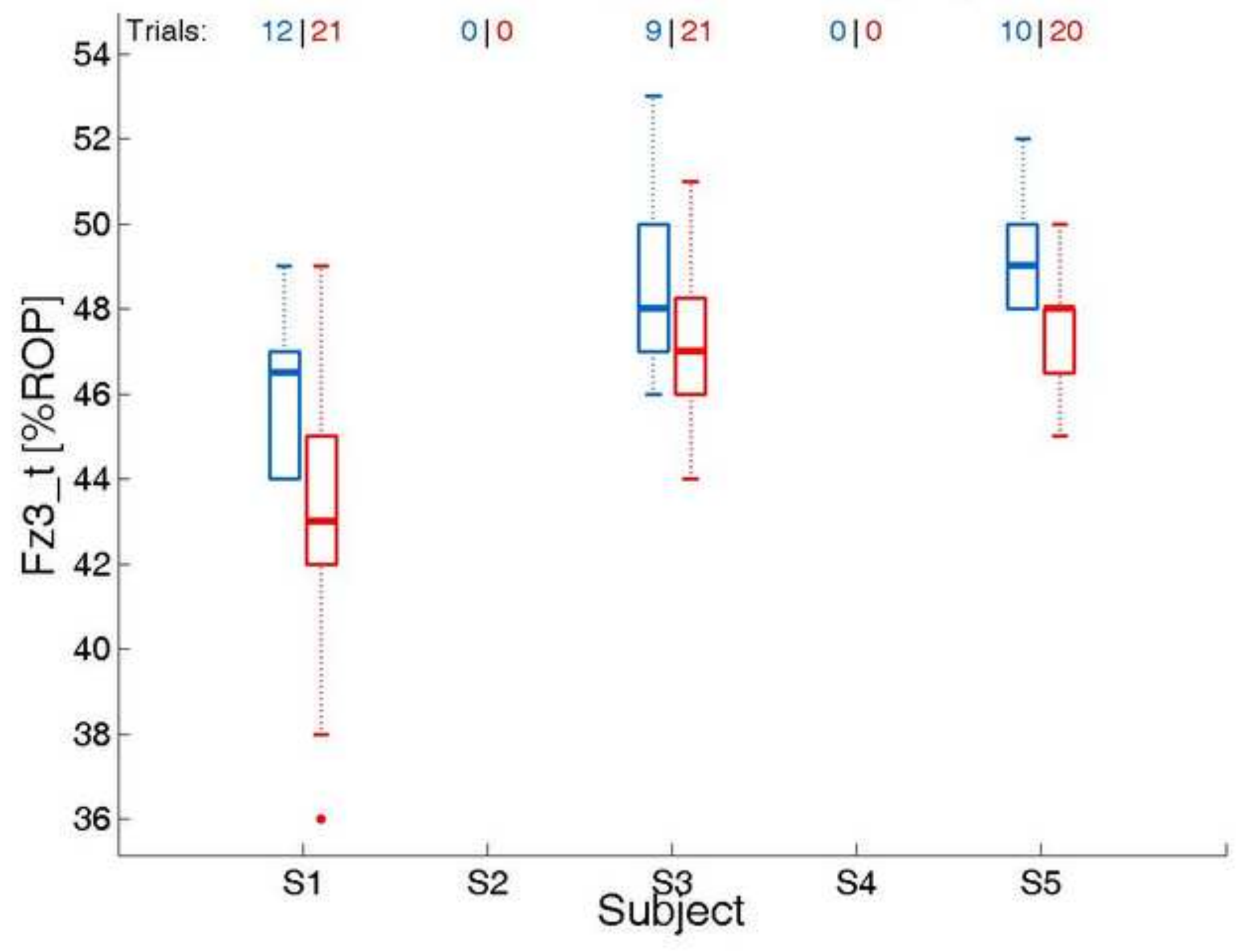


GRF: SKIN (blue) vs. PIN (red)

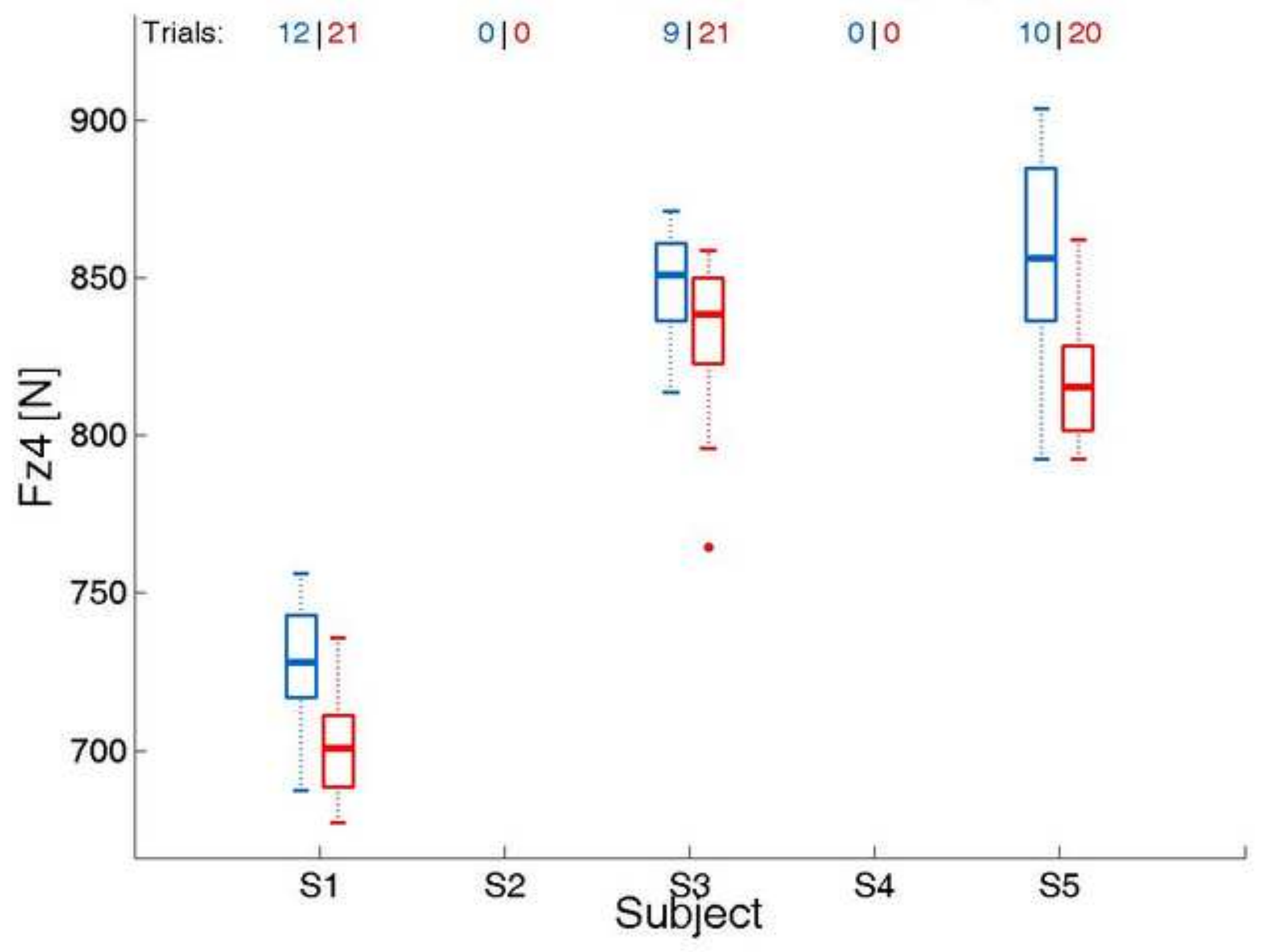


GRF: SKIN (blue) vs. PIN (red)

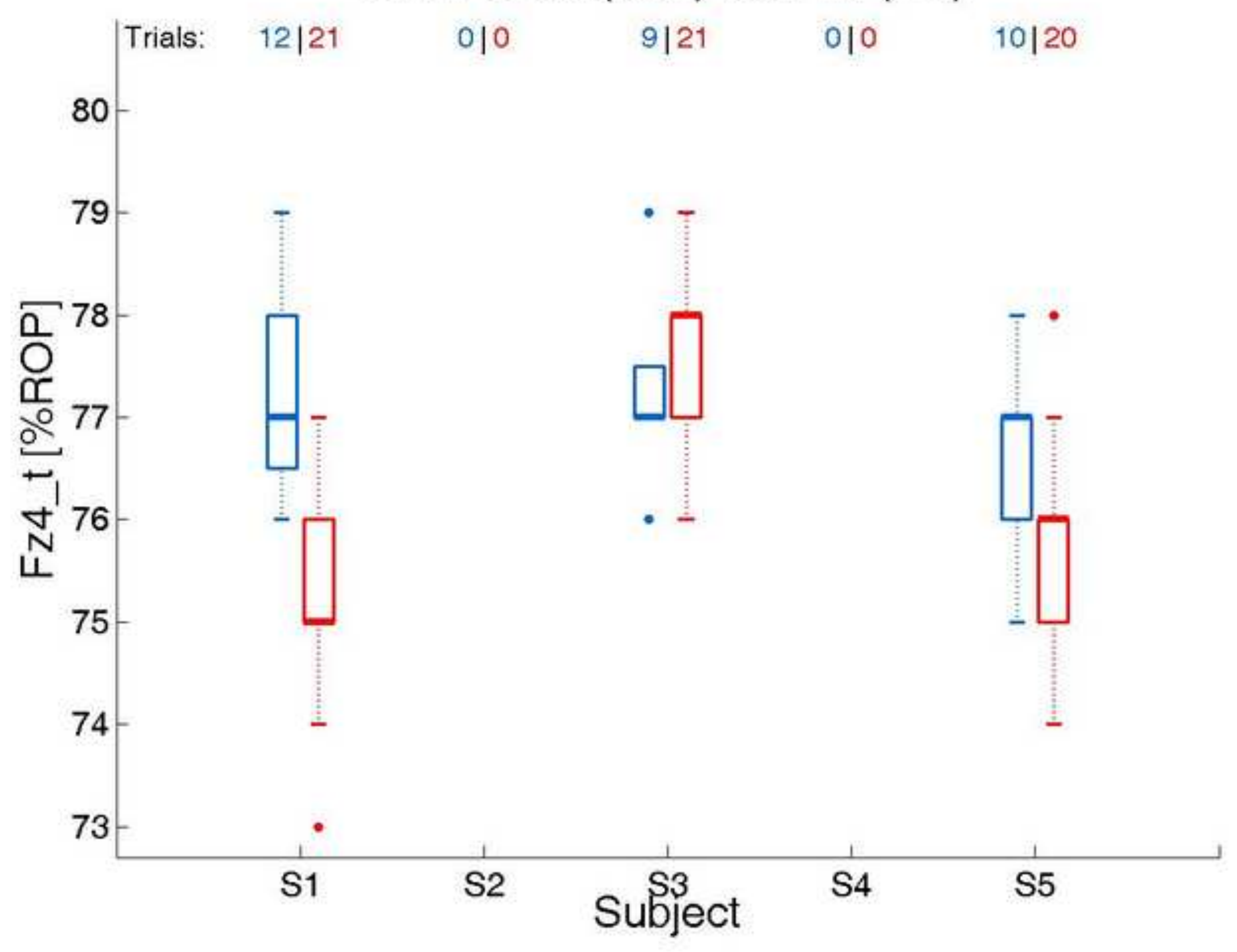




\section{Figure captions}

Figure 1: Marker setup of SKIN and PIN markers involving the foot and tibia.

Figure 2: Division of the plantar pressure signal into 9 subareas as described in [16].

Figure 3: Boxplots of GRF variables. Small numbers above boxplots indicate the number of available trials per subject and condition. 